\title{
GMR
}

\section{Correlation of ERCC1 expression in peripheral blood lymphocytes with outcomes of patients with gastric cancer treated with oxaliplatin-based adjuvant chemotherapy}

\author{
Y.Y. Zhang ${ }^{1}$, K.S. Gu${ }^{1}$, H.Y. Wu ${ }^{1}$, F. Yang ${ }^{2}$, L.J. Bu${ }^{1}$, C.C. Zhao ${ }^{1}$ and \\ Y.R. Zhang ${ }^{1}$ \\ 'Department of Oncology, First Affiliated Hospital of Anhui Medical University, \\ Hefei, Anhui, China \\ 2Department of Pathology, Basic Medicine College of Anhui Medical University, \\ Hefei, Anhui, China \\ Corresponding author: K.S. Gu \\ E-mail:181508706@qq.com \\ Genet. Mol. Res. 14 (4): 15921-15929 (2015) \\ Received June 30, 2015 \\ Accepted September 6, 2015 \\ Published December 7, 2015 \\ DOI http://dx.doi.org/10.4238/2015.December.7.3
}

\begin{abstract}
Excision repair cross-complementing gene-1 (ERCC1) is a key regulatory enzyme whose expression patterns in tumor tissues are associated with survival in gastric cancer. The present study aimed to evaluate the effects of ERCC1 expression in peripheral blood lymphocytes (PBLs) on the outcome of patients with gastric cancer treated with oxaliplatin-based adjuvant chemotherapy. Tumor and PBL samples from 48 patients treated with adjuvant oxaliplatin-based chemotherapy for gastric cancer were analyzed. Immunohistochemistry was used to assess the expression of ERCC1. After a median follow-up of 18.5 months, the median disease-free survival (DFS) and overall survival (OS) were 12 and 20 months, respectively. Expression of ERCC1 was found in $72.9 \%$ (35/48), 56.3\% (27/48), and 10.0\% (2/20) of tumor tissues, PBLs from gastric cancer patients, and PBLs from controls, respectively. A significant
\end{abstract}


positive correlation between ERCC1 expression in PBL and cancer tissue was found $\left(\chi^{2}=12.098, P=0.001\right.$, Pearson contingency coefficient $=$ 0.502). Patients with negative expression of ERCC1 in tumor tissues had a significantly longer median DFS and median OS compared to patients with positive expression of ERCC1 (median DFS, 18 vs 10 months, $\mathrm{P}=0.006$; median OS, 30 vs 17 months, $\mathrm{P}=0.012$ ). In PBLs, high expression of ERCC1 was associated with decreased DFS (9 vs 18 months, $P=0.032$ ), but not OS (16 vs 24 months, $P=0.057$ ). Patients with gastric cancer exhibiting negative expression of ERCC1 are more likely to benefit from oxaliplatin-based adjuvant chemotherapy.

Key words: Stomach neoplasms; Human ERCC1 protein; Oxaliplatin; Adjuvant chemotherapy; Cancer survival

\section{INTRODUCTION}

Gastric cancer is one of the most frequent cancers worldwide and is the second leading cause of cancer death in China. At present, surgery remains the mainstay of cure for patients without distant metastases. However, even following radical surgery, the majority of patients develop recurrence (Macdonald, 2004). Several postoperative adjuvant trials have demonstrated that chemotherapy can have significant benefit for these patients (Carrato et al., 2005), but treatment is still not individualized. It is important that physicians avoid unnecessary toxicity and hospitalization with the aim of preserving economic and human resources. Therefore, the identification of molecular markers that are able to predict outcomes is essential for the development of adjuvant chemotherapy in patients with gastric cancer.

Platinum has significant activity as chemotherapy for gastric cancer. Among platinumcontaining agents, oxaliplatinis a third generation platinum compound. Beneficial results of platinumbased adjuvant chemotherapy were recently seen in two large randomized trials. In the CLASSIC trial, an improvement of $15 \%$ was seen in the 3 -year disease-free survival (DFS) of patients treated with adjuvant oxaliplatin and capecitabine compared to surgery alone (hazard ratio, 0.56; $P<0.0001$ ) (Bang et al., 2012). Moreover, in the ARTIST study, the addition of capecitabine plus cisplatin (XP) plus radiotherapy to XP chemotherapy did not significantly reduce the recurrence rate after curative resection and D2 lymph node dissection in gastric cancer. However, in view of the good efficacy and safety of XP chemotherapy, XP was recommended for postoperative adjuvant chemotherapy (Lee et al., 2012).

Platinum causes the formation of platinum-DNA adducts that block transcription, leading to cytotoxicity and cell death. Studies have shown that cell amplification in response to DNA repair plays an important role in resistance to platinum drugs. Nucleotide excision repair is one of several DNA repair pathways that correct damaged DNA structures (Rabik and Dolan, 2007). Excision repair cross-complementing gene-1 (ERCC1) is a critical enzyme in the nucleotide excision repair pathway (Niedernhofer et al., 2004). As shown in several studies, both ERCC1 protein and mRNA expression are negatively correlated with the efficacy of platinum-based chemotherapy in patients with gastric cancer (Matsubara et al., 2008; Ozkan et al., 2010). In particular, in two recent studies of patients who received cisplatin-based adjuvant chemotherapy after curative resection, high ERCC1 expression was associated with decreased overall survival (OS) (Fareed et al., 2010; Kim et al., 2011). 
However, analyses of ERCC1 expression have been mainly based on tumor tissue samples from operative resection or gastroscopy, which are associated with significant patient discomfort. Therefore, there is a need to develop affordable and non-invasive methods that can detect ERCC1 expression. Many studies have confirmed that peripheral blood lymphocytes (PBL) and tumor cells carry a homologous ERCC1 gene (Kaspers et al., 1991; Yang et al., 2006). In a previous study, we demonstrated that the expression of ERCC1 in PBLs might indirectly reflect ERCC1 expression in gastric cancer tissues (Zhang and Gu, 2013). If the effects of ERCC1 expression in PBL on outcome are confirmed, this could greatly advance clinical practice.

\section{MATERIAL AND METHODS}

\section{Patients and treatments}

Tumor specimens were collected from 48 patients with stage II-IV gastric cancer (AJCC 7th edition) who were recruited during the period from April 2010 to November 2012 and who underwent radical surgery at the First Affiliated Hospital of Anhui Medical University. Inclusion criteria were as follows: 1) histologically confirmed gastric cancer; 2) no previous chemotherapy or radiotherapy; 3 ) availability of clinical and pathological data; 4) good organ functions; 5) signed informed consent prior to entering the study; 6) no serious liver, kidney, pulmonary, or cardiovascular diseases; 7) Eastern Cooperative Oncology Group performance status (ECOG-PS) <3; 8) no prior palliative resection of advanced metastatic disease; and 9) no obvious signs of recurrence with imaging at baseline assessment after surgery.

The study protocol was approved by the Institutional Ethics Committee of Anhui Medical University and was conducted according to the principles of the Declaration of Helsinki. All patients had signed an informed consent before enrollment.

Adjuvant chemotherapy consisted in 4-6 courses of XELOX, administered as follows: oxaliplatin (100 mg/m² D1) and capecitabine (1000 mg/m²BID D1-14) every 3 weeks.

In addition, peripheral blood from 20 non-cancer patients was collected as a control group.

\section{Follow-up evaluation and endpoints}

Results from physical examination, hematological studies, and whole-body computed tomography as well as the carcinoembryonic antigen levels were assessed every 3 months in the first year and every 6 months thereafter. Patients underwent gastroscopy every 12 months after surgery. Recurrences were documented by clinical or radiologic assessment. Follow-up was closed on May 2014.

Analyses for OS and DFS were performed. OS was the time-period from study initiation until the date of mortality, regardless of cause, or until the most recently documented follow-up. DFS was calculated from time of diagnosis to disease recurrence or last follow-up.

\section{Separation of PBL}

Peripheral blood samples were collected in heparin sodium-containing tubes from a control group and from gastric cancer patients before chemotherapy. PBLs were isolated from the whole blood on histopaque gradients (Histopaque 1077, TBD, Tianjin, China). 


\section{Immunohistochemistry}

Formalin-fixed, paraffin-embedded tumor specimens obtained by biopsy were cut into 4- $\mu \mathrm{m}$ sections. Slides were deparaffinized in xylene for epitope retrieval, exposed to $10 \mathrm{mM}$ citric acid antigen retrieval solution ( $\mathrm{pH}$ 6.0), and heated for $30 \mathrm{~min}$ in a water bath. PBLs were fixed with methanol and Triton X-100 was used to permeabilize membranes.

Specimens were incubated overnight with a monoclonal antibody against human ERCC1 protein (mouseclone 8F1, Maixin Bio, Fujian, China). Antibody binding was detected with the Maxvision kit (sheep/rabbit anti-mouse, Maixin Bio, Fujian, China) by incubation for 15 min with the substrate and counterstained with hematoxylin.

Two pathologists who were unaware of clinical data independently evaluated the percentage of positive tumor nuclei under a light microscope at a magnification of $400 \mathrm{X}$. The grading system was as follows: immunoreactivityin $\geq 10 \%$ of tumor cells or PBLs was considered as positive. If $<10 \%$, it was defined as negative (Wachters et al., 2005).

\section{Statistical analysis}

Data analysis was performed using SPSS 18.0 for Windows (SPSS Inc., Chicago, USA). Statistical significance was defined as $\mathrm{P}<0.05$. The association of ERCC1 expression with clinicopathological features was assessed using chi-square analysis. The strength of correlation between ERCC1 expression in tumor tissue and PBLs was assessed with the Pearson correlation test. Kaplan-Meier log-rank survival analysis was performed to evaluate the association of expression of ERCC1 with survival.

\section{RESULTS}

\section{Patient characteristics}

Demographic and pathologic characteristics are summarized in Table 1. The cohort included 30 men $(62.5 \%)$ and 18 women (37.5\%), with a median age of 59 years (range: 2477 years). AJCC staging yielded 19 stage II cases, and 29 stage III or IV cases. After surgery, all patients received adjuvant treatment. At the end of follow-up, 44 patients experienced tumor recurrence or distant metastasis, and 33 patients died of gastric cancer related complications. Disease relapse accounted for all deaths (33 events). After a median follow-up of 18.5 months (range 3-47), DFS and OS were $12(95 \% \mathrm{Cl}=8.982-15.018)$ and $20(95 \% \mathrm{Cl}=15.504-24.496)$ months, respectively.

\section{Expression of ERCC1}

ERCC1 expression was positive in $72.9 \%$ (35/48), 56.3\% (27/48), and $10.0 \%(2 / 20)$ of tumor tissues, PBLs from gastric cancer patients, and PBLs in the control group, respectively (Figure 1). A significant positive correlation between ERCC1 expression in PBL and cancer tissue was found $\left(\chi^{2}=12.098, P=0.001\right.$, Pearson contingency coefficient $\left.=0.502\right)$. There was no relationship between ERCC1 expression and clinicopathological parameters including age, sex, clinical stage, T stage, tumor location, presence of lymph node metastasis, or degree of differentiation (Table 1). 


\begin{tabular}{|c|c|c|c|c|c|c|}
\hline Characteristics & Number & $(\%)$ & ERCC1-positive cases in PBLs & $\chi^{2}(\mathrm{P})$ & ERCC1-positive cases in tumor tissues & $\chi^{2}(P)$ \\
\hline \multicolumn{7}{|l|}{ Age (years) } \\
\hline$\geq 59$ & 26 & 54.2 & 19 & 0.978 & 14 & 0.715 \\
\hline$<59$ & 22 & 45.8 & 16 & $(0.001)$ & 13 & $(0.133)$ \\
\hline \multicolumn{7}{|l|}{ Gender } \\
\hline Male & 30 & 62.5 & 20 & 0.851 & 16 & 0.277 \\
\hline Female & 18 & 37.5 & 15 & $(0.356)$ & 11 & $(0.599)$ \\
\hline \multicolumn{7}{|l|}{ Stage } \\
\hline II & 19 & 39.6 & 15 & 0.579 & 11 & 0.035 \\
\hline III + IV & 29 & 60.4 & 20 & $(0.447)$ & 16 & $(0.853)$ \\
\hline \multicolumn{7}{|l|}{ T stage } \\
\hline $\mathrm{T} 1+\mathrm{T} 2$ & 9 & 18.8 & 9 & 2.599 & 7 & 1.148 \\
\hline $\mathrm{T} 3+\mathrm{T} 4$ & 39 & 81.3 & 26 & $(0.107)$ & 20 & $(0.284)$ \\
\hline \multicolumn{7}{|l|}{ Tumor location } \\
\hline Distal & 27 & 56.3 & 20 & 0.042 & 16 & 0.227 \\
\hline Proximal & 21 & 43.8 & 15 & $(0.838)$ & 11 & $(0.634)$ \\
\hline Lymph node metastasis & $0-24$ & & & & & \\
\hline Yes & 35 & 72.9 & 25 & 0.000 & 19 & 0.203 \\
\hline No & 13 & 27.1 & 10 & $(0.988)$ & 8 & $(0.653)$ \\
\hline \multicolumn{7}{|l|}{ Differentiation } \\
\hline Poor & 25 & 52.1 & 16 & 2.101 & 13 & 0.383 \\
\hline Moderate or well & 23 & 47.9 & 19 & $(0.147)$ & 14 & $(0.536)$ \\
\hline
\end{tabular}

ERCC1 = excision repair cross-complementing gene-1; PBL = peripheral blood lymphocyte.
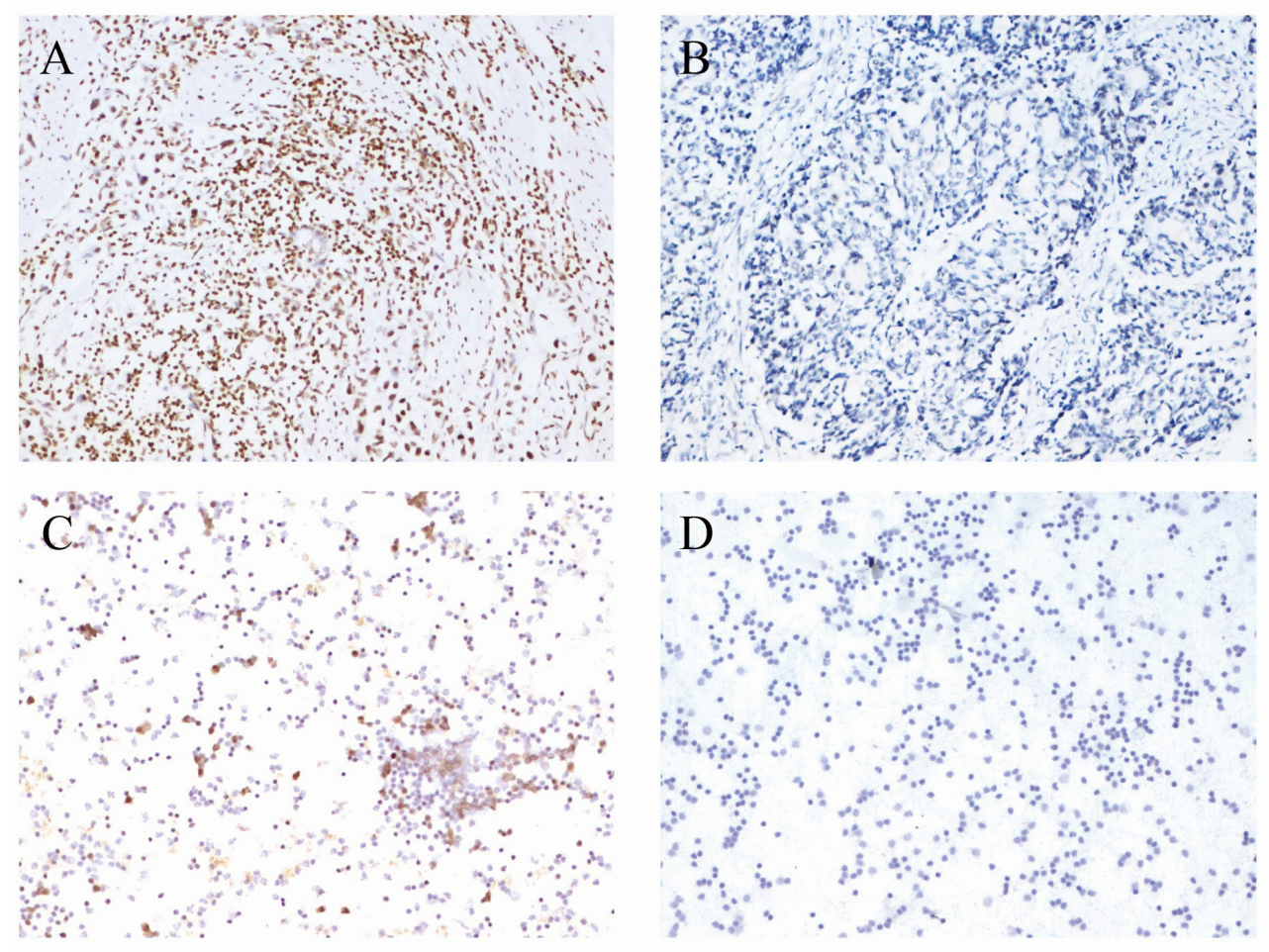

Figure 1. Staining of ERCC1 showing positive immunoreactivity in tumor tissue (A) and PBLs (C). ERCC1 with negative immunoreactivity in tumor tissue (B) and PBLs (D). (All figures, 100X). ERCC1 = excision repair cross-complementing gene-1; PBL = peripheral blood lymphocyte. 


\section{ERCC1 and clinical outcomes}

Patients with negative expression of ERCC1 in tumor tissues had significantly longer median DFS and median OS compared to patients with positive expression of ERCC1 (median DFS, 18 vs 10 months, $\mathrm{P}=0.006$; median OS, 30 vs 17 months, $\mathrm{P}=0.012$, respectively) (Figure 2).
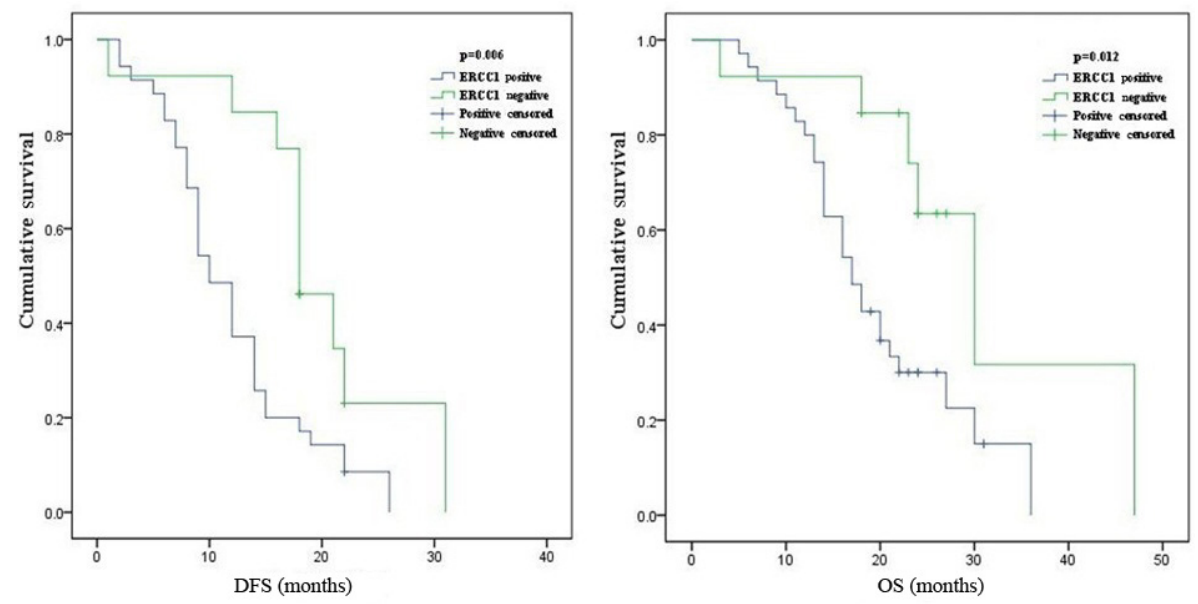

Figure 2. Kaplan-Meier estimates of DFS and OS according to ERCC1 expression in tumor tissues. DFS = diseasefree survival; OS = overall survival; ERCC1 = excision repair cross-complementing gene-1.

Moreover, in PBLs from patients with gastric cancer, positive expression of ERCC1 was associated with significantly decreased DFS ( 9 vs 18 months, $P=0.032$ ). However, positive expression of ERCC1 was not associated with OS (16 vs 24 months, $\mathrm{P}=0.057$ ) (Figure 3 ).
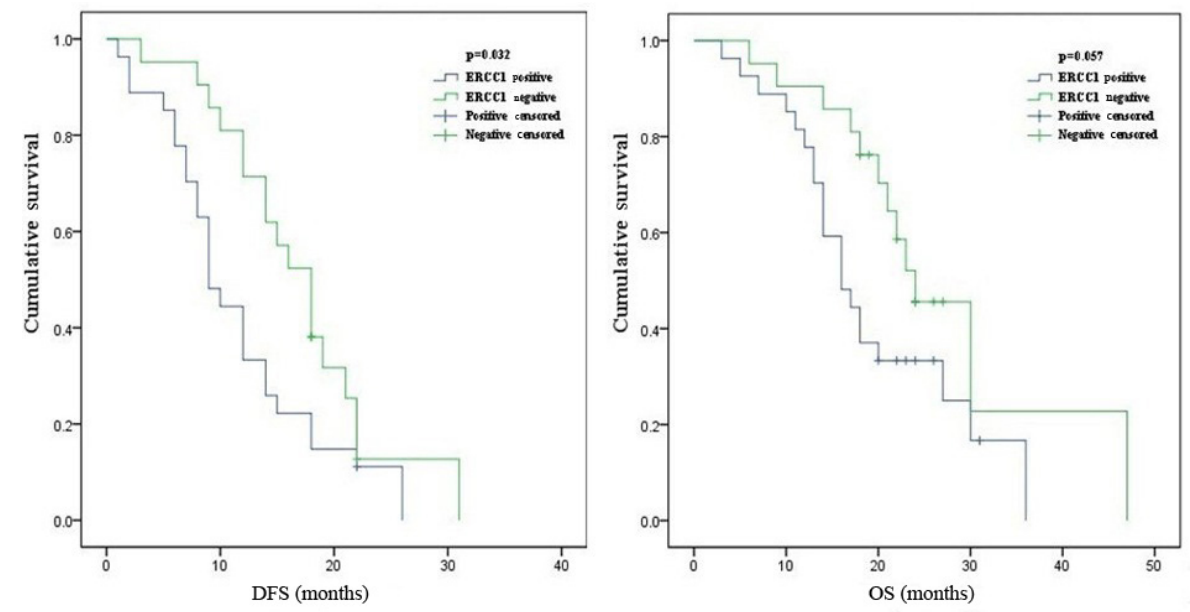

Figure 3. Kaplan-Meier estimates of DFS and OS according to ERCC1 expression in PBLs. DFS = disease-free survival; OS = overall survival; ERCC1 = excision repair cross-complementing gene-1. 


\section{DISCUSSION}

Given the high rates of recurrence and poor survival associated with resected gastric cancer, molecular biomarkers that can guide treatment decisions are needed. Platinum-based adjuvant chemotherapy remains the backbone of therapy in gastric cancer. Oxaliplatin, a third generation platinum analog, is widely used in this regard. Research has shown that the mechanisms of resistance of tumor to platinum drugs include reduction of dosage accumulation, enhanced tolerance of platinum-DNA complexes, and enhancement of DNA repair. It is worth noting that ERCC1 plays an important role in the enhancement of DNA repair (Metzger et al., 1998).

Many clinical studies have extensively investigated the association between ERCC1 expression and outcomes after adjuvant chemotherapy in gastric cancer. In the study by Squires et al., 109 patients who underwent resection for gastric cancer had tissue available for analysis of expression of ERCC1 using immunohistochemistry. High tumoral expression of ERCC1 was associated with decreased OS in patients who received platinum-based adjuvant chemotherapy (16.7 months vs 53.8 months; $\mathrm{P}=0.03$ ) (Squires et al., 2013). In the investigation by De Dosso et al. (2013), the expression of ERCC1 expression in tumor samples obtained from surgical specimens of 68 patients treated with adjuvant cisplatin-based chemotherapy for gastric cancer were analyzed by immunohistochemistry. Significantly longer OS $(P=0.04)$ was found in patients categorized as ERCC1 negative by immunohistochemistry. Thus, ERCC1 may help in predicting which patients are more likely to benefit from adjuvant cisplatin-based chemotherapy in curatively resected gastric cancer (De Dosso et al., 2013). In a study from China, the effects of ERCC1 expression on survival in were evaluated in 57 gastric cancer patients treated with surgery followed by oxaliplatin-based adjuvant chemotherapy. ERCC1 expression levels were measured by using qRT-PCR. Univariate analyses revealed that patients with low ERCC1 levels had longer relapse-free survival (RFS) and OS than those with high ERCC1 levels (median RFS, 18 vs 7 months, $\mathrm{P}=0.001$; median OS, 27 vs 11months, $P=0.001)$. It was concluded that gastric cancer patients with low levels of ERCC1 expression demonstrated a benefit from oxaliplatin-based adjuvant chemotherapy (Liu et al., 2013). In our study, the association between ERCC1 expression in tumor tissues and outcome in resected patients treated with oxaliplatin-based regimens confirms what has been described by other authors. Patients with negative expression of ERCC1 had a significantly longer median DFS and median OS compared with patients with positive expression of ERCC1 (median DFS, 18 vs 10 months, $\mathrm{P}=0.006$; median OS, 30 vs 17 months, $\mathrm{P}=0.012$, respectively).

However, analyses of ERCC1 expression have been mainly based on primary tumor samples, and obtaining them is associated with substantial patient discomfort. Moreover, detection of ERCC1 expression using tumor tissue is troublesome. Thus, a simple method of detection is desirable for routine clinical practice.

Several studies have confirmed that both PBL and tumor cells carry a homologous gene for ERCC1 (Kaspers et al., 1991; Yang et al., 2006). In patients with non-small cell lung cancer and those with head and neck squamous cell carcinoma, the levels of ERCC1 in PBLs may be considered prognostic (Schena et al., 2012). In a previous study, we also detected ERCC1 expression in 53 gastric cancer patients in both tumor tissue and PBLs. We found that the ERCC1 expression in PBLs indirectly reflected ERCC1 expression in cancer tissues (Zhang and Gu, 2013).

However, it remains unclear if ERCC1 expression in PBLs predicts outcome in patients with gastric cancer treated with platinum-based chemotherapy. In epithelial ovarian cancer, Darcy et al. (2007) analyzed whether mRNA expression of ERCC1 in PBLs was associated with clinical 
outcomes in patients treated with platinum-taxane chemotherapy, although negative results were reported. Herein, we assessed correlations between expression of ERCC1 in PBLs and outcomes in 48 gastric cancer patients treated with oxaliplatin-based adjuvant chemotherapy. We found that positive ERCC1 expression was associated with significantly decreased DFS (9 vs 18 months, $\mathrm{P}=0.032$ ). However, positive ERCC1 expression was not associated with OS (16 vs 24 months, $\mathrm{P}=0.057$ ).

In addition, there are some potential limitations of our study. First, the sample size was relatively small, especially for survival analyses. In addition, the follow-up time was relatively short, although it was problematic to obtain OS in all patients. Lastly, the potential effect that platinumbased chemotherapy may have on the expression of ERCC1 in tumor tissue and PBL was clear. However, after chemotherapy is administered, repeated detection of ERCC1 expression using tumor tissue is troublesome. For this reason, repeated detection of ERCC1 expression in PBL may be helpful in individualized treatment. Therefore, if ERCC1 expression in PBL was detected during the entire course of chemotherapy, it could reveal resistance to platinum-based chemotherapy in gastric cancer in a timely manner.

In conclusion, we demonstrate that patients with negative ERCC1 expression are more likely to benefit from oxaliplatin-based adjuvant chemotherapy. To further confirm the prognostic value of ERCC1 expression of tumor tissue and PBLs in gastric cancer, a multicenter prospective study with a large sample size is required.

\section{ACKNOWLEDGMENTS}

We thank everyone at our institution who helped with this study. Research supported by grants from the Anhui Provincial Natural Science Foundation (\#1408085MH203).

\section{REFERENCES}

Bang YJ, Kim YW, Yang HK, Chung HC, et al. (2012). Adjuvant capecitabine and oxaliplatin for gastric cancer after D2 gastrectomy (CLASSIC): a phase 3 open-label, randomised controlled trial. Lancet 379: 315-321.

Carrato A, Gallego-Plazas J and Guillen-Ponce C (2005). Adjuvant therapy of resected gastric cancer is necessary. Semin. Oncol. 32: 105-108.

Darcy KM, Tian C and Reed E (2007). A Gynecologic Oncology Group study of platinum-DNA adducts and excision repair cross-complementation group 1 expression in optimal, stage III epithelial ovarian cancer treated with platinum-taxane chemotherapy. Cancer Res. 67: 4474-4481.

De Dosso S, Zanellato E, Nucifora M, Boldorini R, et al. (2013). ERCC1 predicts outcome in patients with gastric cancer treated with adjuvant cisplatin-based chemotherapy. Cancer Chemother. Pharmacol. 72: 159-165.

Fareed KR, Al-Attar A, Soomro IN, Kaye PV, et al. (2010). Tumour regression and ERCC1 nuclear protein expression predict clinical outcome in patients with gastro-oesophageal cancer treated with neoadjuvant chemotherapy. Br. J. Cancer 102: 1600-1607.

Kaspers CIL, Pieters R, Zantwijk CHV, De Laat PA, et al. (1991). In vitro drug sensitivity of normal peripheral blood lymphocyte and childhood leukaemic from bone marrow and peripheral blood. Br. J. Cancer 64: 469-474.

Kim KH, Kwon HC, Oh SY, Kim SH, et al. (2011). Clinicopathologic significance of ERCC1, thymidylate synthase and glutathione S-transferase P1 expression for advanced gastric cancer patients receiving adjuvant 5-FU and cisplatin chemotherapy. Biomarkers 16: 74-82.

Lee J, Lim do H, Kim S, Park SH, et al. (2012). Phase III trial comparing capecitabine plus cisplatin versus capecitabine plus cisplatin with concurrent capecitabine radio therapy in completely resected gastric cancer with D2 lymph node dissection: the ARTIST trial. J. Clin. Oncol. 30: 268-273.

Liu YP, Ling Y, Qi QF, Zhang YP, et al. (2013). The effects of ERCC1 expression levels on the chemosensitivity of gastric cancer cells to platinum agents and survival in gastric cancer patients treated with oxaliplatin-based adjuvant chemotherapy. Oncol. Lett. 5: 935-942. 
Macdonald JS (2004). Treatment of localized gastric cancer. Semin. Oncol. 31: 566-573.

Matsubara J, Nishina T, Yamada Y, Moriwaka T, et al. (2008). Impacts of excision repair cross-complementing gene-1 (ERCC1), dihydropyrimidine dehydrogenase, and epidermal growth factor receptor on the outcomes of patients with advanced gastric cancer. Br. J. Cancer 98: 832-839.

Metzger R, Leichman CG, Danenberg KD, Danenberg PV, et al. (1998). ERCC1 mRNA levels complement thymidylate synthase mRNA levels in predicting response and survival for gastric cancer patients receiving combination cisplatin and fluorouracil chemotherapy. J. Clin. Oncol. 16: 309-317.

Niedernhofer LJ, Odijk H, Budzowska M, van Drunen E, et al. (2004). The structure specific endonuclease ERCC1 XPF is required to resolve DNA interstrand cross-link induced double strand breaks. Mol. Cell Biol. 24: 5776-5787.

Ozkan M, Akbudak IH, Deniz K, Dikilitas M, et al. (2010). Prognostic value of excision repair cross-complementing gene 1 expression for cisplatin-based chemotherapy in advanced gastric cancer. Asian Pac. J. Cancer Prev. 11: 181-185.

Rabik CA and Dolan ME (2007). Molecular mechanisms of resistance and toxicity associated with platinating agents. Cancer Treat. Rev. 33: 9-23.

Schena M, Guarrera S, Buffoni L, Salvadori A, et al. (2012). DNA repair gene expression level in peripheral blood and tumour tissue from non-small cell lung cancer and head and neck squamous cell cancer patients. DNA Repair 11: 374-380.

Squires MH 3rd, Fisher SB, Fisher KE, Patel SH, et al. (2013). Differential expression and prognostic value of ERCC1 and thymidylate synthase in resected gastric adenocarcinoma. Cancer 119: 3242-3250.

Wachters FM, Wong LS, Timens W, Kampinga HH, et al. (2005). ERCC1, hRad51, and BRCA1 protein expression in relation to tumor response and survival of stage III/IVNSCLC patients treated with chemotherapy. Lung Cancer 50: 211-219.

Yang M, Kim WH, Choi Y, Lee SH, et al. (2006). Effects of ERCC1 expression in peripheral blood on the risk of head and neck cancer. Eur. J. Cancer Prev. 15: 269-273.

Zhang YY and Gu KS (2013). DNA repair capacity in peripheral blood lymphocytes predicts efficacy of platinum-based chemotherapy in patients with gastric cancer. Asian Pac. J. Cancer Prev. 14: 5507-5512. 